\title{
DOUBLE FREE-FLAP RECONSTRUCTION OF MASSIVE DEFECTS INVOLVING THE LIP, CHIN, AND MANDIBLE
}

\author{
WILLIAM M. KUZON, JR., M.D., Ph.D., ${ }^{1 *}$ \\ SAMEER JEJURIKAR, M.D., ${ }^{1}$ EDWIN G. WILKINS, M.D., ${ }^{1}$ and \\ WILLIAM M. SWARTZ, M.D. ${ }^{2}$
}

Two patients with massive, composite defects of the total lower lip, chin, and anterior mandible underwent double freeflap reconstruction. A fibular osteoseptocutaneous flap was used to reconstruct the mandible and floor of the mouth and a radial forearm fasciocutaneous composite flap, including the palmaris longus tendon, was used for total lower lip and chin reconstruction. Postoperatively, both patients had acceptable cosmesis, were orally competent, and recovered adequate mandibular function. Double free-flap reconstruction is indicated only in those circumstances in which composite tissue requirements or massive tissue defects preclude reconstruction with a single free-tissue transfer. (c) 1998 Wiley-Liss, Inc.
18:372-378 1998
Over the past 25 years, microvascular tissue transfers have revolutionized the reconstruction of major defects of the head and neck, becoming the method of choice for most patients. ${ }^{1}$ Although most head and neck defects can be reconstructed using a single free-tissue transfer, the use of two or even three free flaps is indicated for massive tissue defects or for composite tissue requirements that cannot be met with a single free flap. ${ }^{2}$ Two patients with composite defects of the central mandible, entire lower lip, and entire chin underwent single-stage, double free-flap reconstruction utilizing a fibular osteoseptocutaneous flap to reconstruct the mandible and floor of the mouth, ${ }^{3-5}$ as well as an innervated radial forearm fasciocutaneous composite flap, including palmaris longus tendon, for total lower lip and chin reconstruction. ${ }^{6}$ Both patients had acceptable functional and aesthetic outcomes.

\section{CASE REPORTS}

\section{Case 1}

A 61-year-old Caucasian male presented with a lesion involving his lower lip and chin (Fig. 1A). Physical examination revealed a $3-\mathrm{cm}$ mass on the lower lip and an $8-\mathrm{cm}$ firm lesion fixed to the mandible in the area of the right parasymphysis. Biopsy of the lower lip lesion confirmed a diagnosis of squamous cell carcinoma. Clinical examination, direct laryngoscopy, and a high-resolution CT scan of

\footnotetext{
${ }^{1}$ Department of Surgery, University of Michigan, Ann Arbor, MI ${ }^{2}$ Department of Surgery, University of Pittsburgh, Pittsburgh, PA
}

${ }^{*}$ Correspondence to: William M. Kuzon, Jr., M.D., Ph.D., Plastic and Recon structive Surgery, 2130 Taubman Health Care Center, 1500 East Medical Center Drive, Ann Arbor, Ml 48109-0340. E-mail: wkuzon@umich.edu the mandible and neck determined that the chin mass invaded the outer cortex of the mandible and that no nodal metastasis or synchronous primaries existed. Full-body CT scan to evaluate for distant metastasis was negative; the tumor was staged as T4N0M0. The institutional, multidisciplinary tumor board recommended surgical resection followed by reconstruction and postoperative radiation therapy as the most appropriate treatment. The patient had no other major medical conditions.

The patient underwent a tracheostomy prior to ablative surgery. A composite resection was performed that included a left supraomohyoid neck dissection, a right modified radical neck dissection, and removal of the primary tumor. At the primary site, a composite resection of the lower lip from commissure to commissure, the entire chin, the mandible from midbody on the left to the angle on the right, and a $5.0-\mathrm{cm}$ by $8.0-\mathrm{cm}$ portion of the floor of the mouth anterior to the frenulum of the tongue was performed (Fig. 1B). The external skin defect measured approximately $12.5 \mathrm{~cm}$ by 8.0 $\mathrm{cm}$. Frozen section analyses of the resection margins were negative for residual tumor.

A fibular osteoseptocutaneous flap, with a skin paddle in the distal one-third of the leg centered on strong peroneal artery perforators, was elevated. Prior to resection of the mandible, a reconstruction plate had been contoured and fixed with tapped screws to serve as a guide for in situ closing wedge osteotomies on the fibula and for rigid fixation of the fibula to the mandible (Fig. 1C). After transfer to the mouth, the fibular skin paddle was used to reconstruct the floor of mouth defect and the contoured fibula was used to reconstruct the anterior mandible. The flap was revascularized via anastomoses in the right neck.

A composite radial forearm flap, including the palmaris 

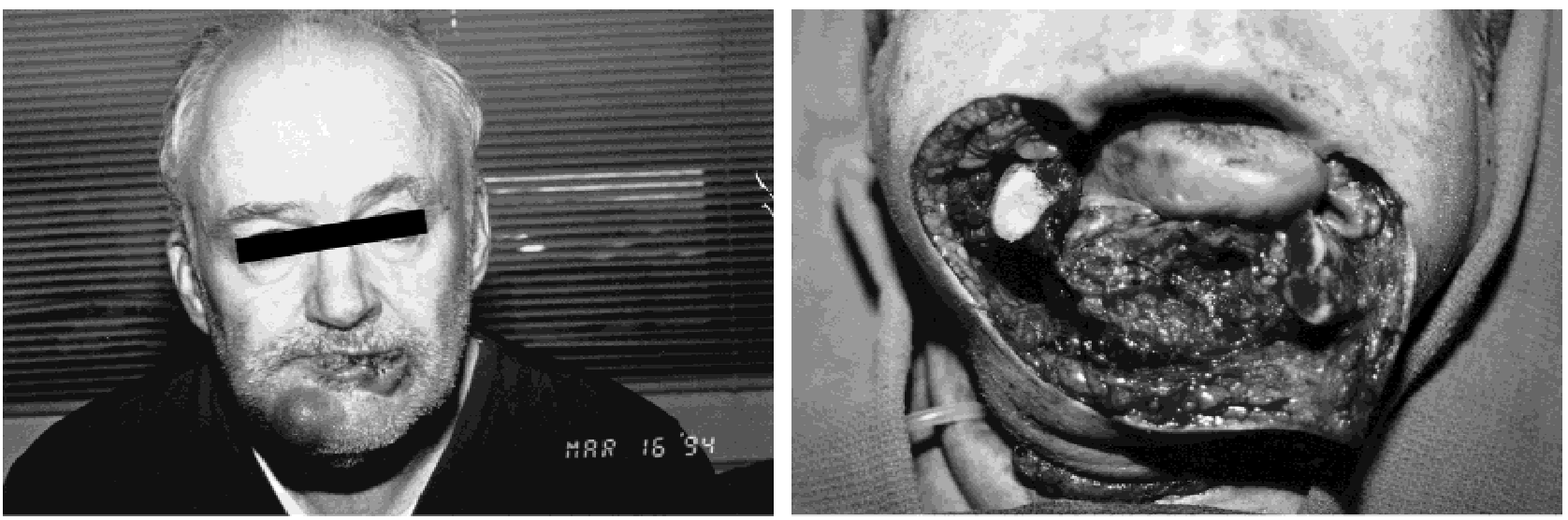

A

B
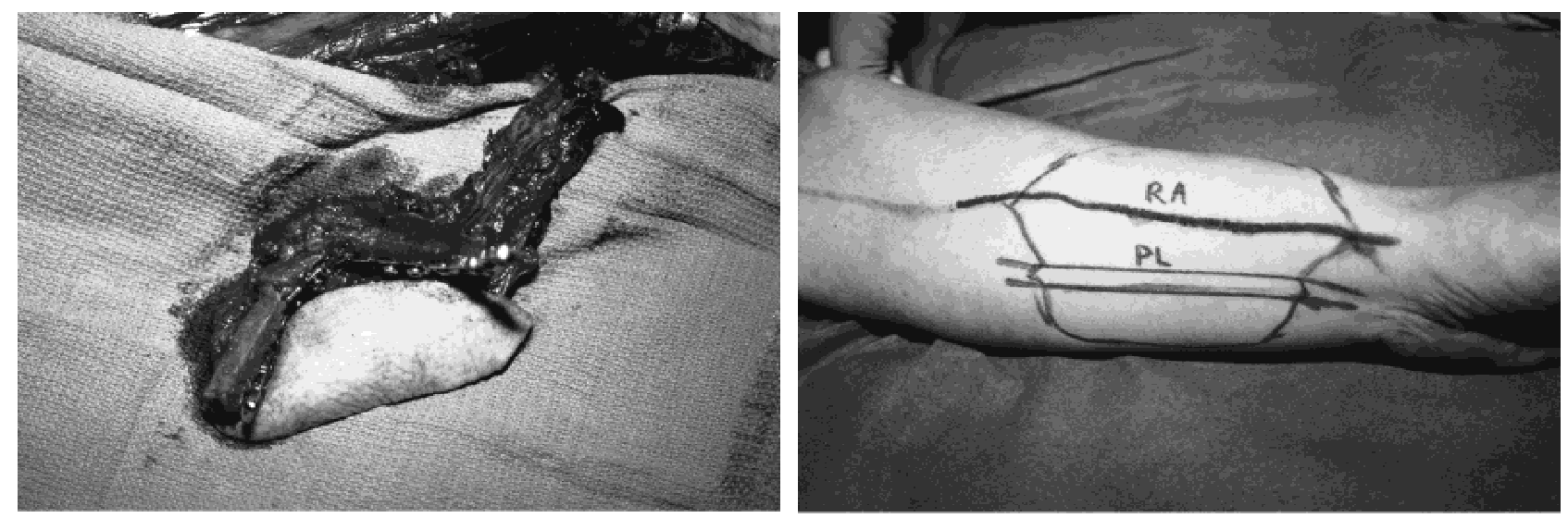

\section{C}

Figure 1. A: Preoperative view of patient 1. Note extensive lesion of left inferior lip and right chin. B: Composite resection included anterior mandible, total lip, total chin, and floor of the mouth. $\boldsymbol{C}$ : In situ closing wedge osteotomies have been performed and the fibula has been secured to a reconstruction plate contoured prior to resection of the mandible. D: Planned radial forearm fasciocutaneous flap including palmaris longus tendon. (Continued on the overleaf.)

longus tendon, was planned and elevated on the left volar forearm so that the cephalic margin of the reconstructed lip was oriented longitudinally along the palmaris longus tendon (Fig. 1D, E). The lateral antebrachial cutaneous nerve was identified and traced proximally to provide for sensory reinnervation of the flap. After transfer to the lower lip, the palmaris longus tendon was sutured to the modioli bilaterally, taking care to maintain moderate tension. The most radial portion of the flap was turned over to provide lining for the inside of the lower lip and buccal sulcus, and was sutured to the most anterior border of the fibular skin paddle, already present intraorally. The ulnar portion of the flap provided external coverage for the lower lip and chin. The flap was revascularized to recipient vessels in the left neck. The flap was not reinnervated because the aveolar nerves were not available for coaptation.

The patient's wounds were closed primarily, without significant tension (Fig. 1F). Ten days postoperatively, he was able to resume oral intake without difficulty. Three weeks postoperatively, the patient was able to undergo a full course of adjuvant radiation therapy. His wounds healed without complications (Fig. 1G, H). The patient was last seen 13 months postoperatively, at which time he was disease free, and had maintained acceptable oral competence and mandibular opening.

\section{Case 2}

A 17-year-old, otherwise healthy female suffered a shotgun blast to the head, resulting in a defect involving the chin, lower lip, floor of the mouth, and mandible from the right ramus to the left midbody. On the day of her injury, a tracheostomy was performed and an external fixator was placed to maintain the position of the lateral mandibular segments. Soft-tissue closure was achieved by advancing neck and cheek skin into the lower lip and chin defect. Her wounds healed uneventfully, and 1 month after injury, the patient was referred for flap reconstruction. A soft-tissue 


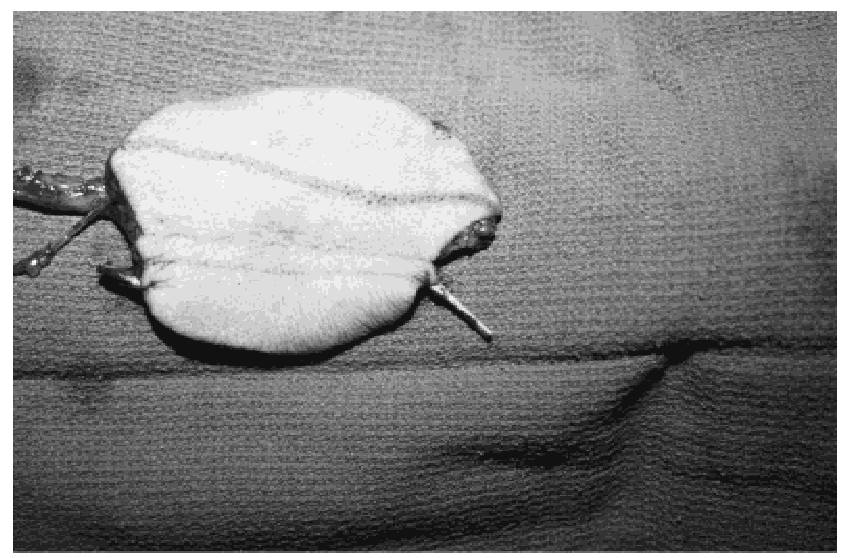

E

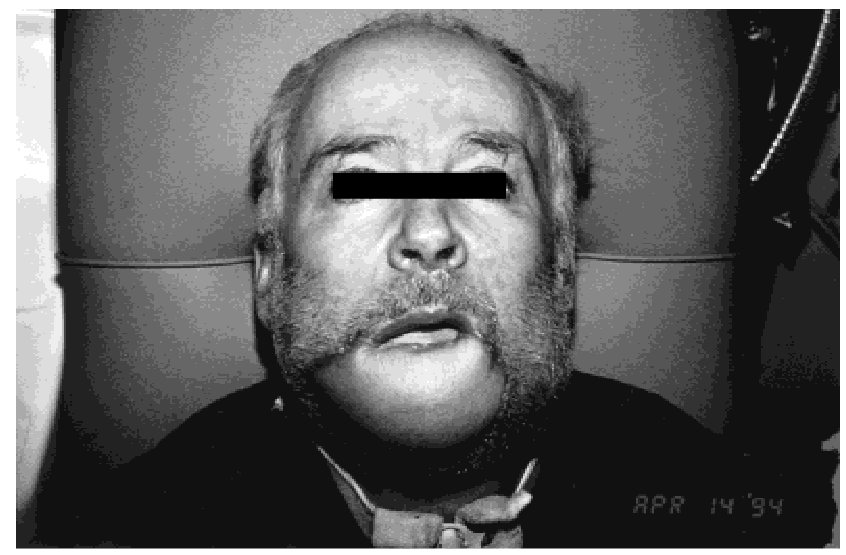

G

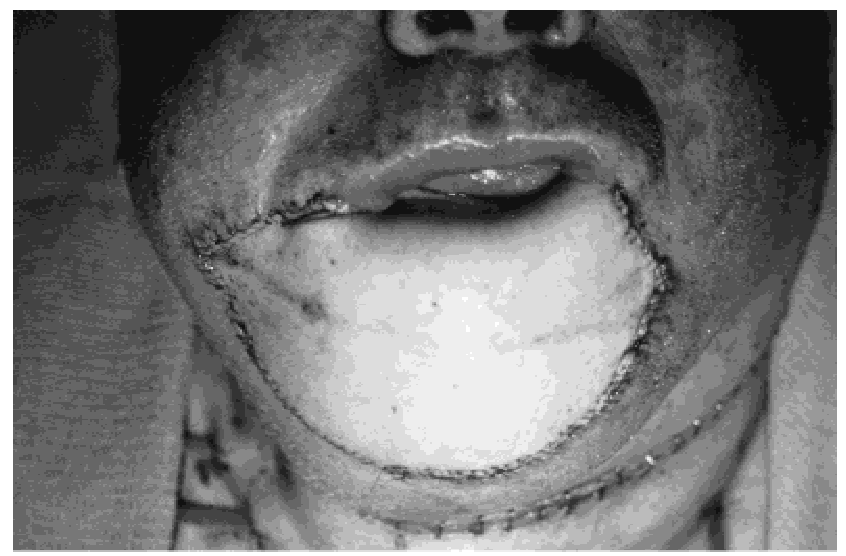

$\mathbf{F}$

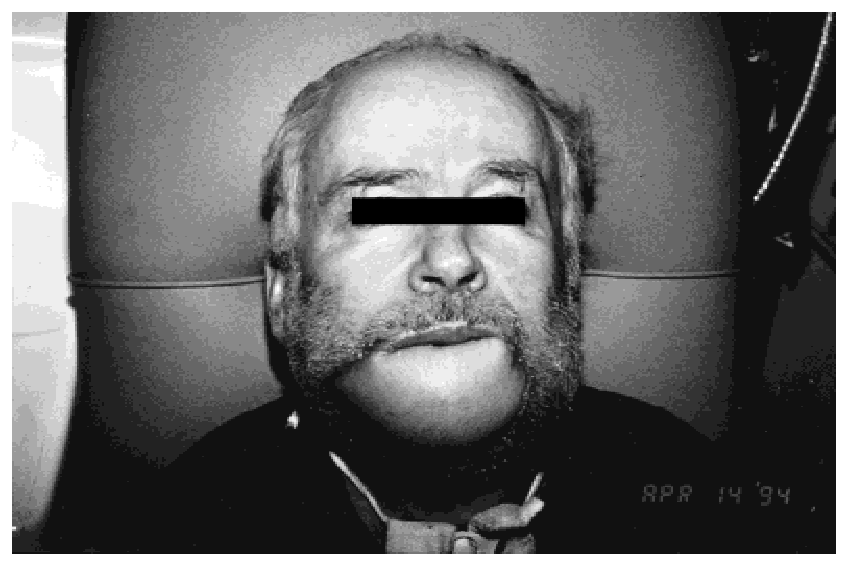

H

Figure 1. (Continued). E: Elevated radial forearm fasciocutaneous flap including palmaris longus tendon. F: Immediate postoperative view. G: Two-week postoperative view. H: Two-week postoperative view; patient is holding air with pursed lips, illustrating oral competence.

defect of the entire lower lip, chin, and floor of mouth was noted in association with the mandibular defect (Fig. 2A, $\mathrm{B})$; reconstructive requirements were identical to those in Case 1.

All previous suture lines within the head and oral cavity were opened, reestablishing the original defect (Fig. 2C). A reconstruction plate was contoured to a cadaveric mandible of similar size and placed to serve as a template for bony restoration without distorting the position of the lateral mandibular segments. A fibular osteoseptocutaneous flap with a skin island measuring $8.0 \mathrm{~cm}$ by $4.0 \mathrm{~cm}$ was elevated. Using the reconstruction plate, closing wedge osteotomies were performed in situ before transfer of the flap to the head and neck. The fibula was secured to the residual mandible using the reconstruction plate and the skin paddle was sutured to the tongue base, filling the floor of the mouth defect. The flap was revascularized using recipient vessels in the right neck.

A composite radial forearm flap including the palmaris longus tendon and the medial and lateral antebrachial cutaneous nerves was elevated and used to reconstruct the lower lip and chin in the same way as for Case 1. The flap was revascularized using the left facial artery and vein. In order to provide sensation to the flap, the lateral antebrachial cutaneous nerve (LABC) was coapted to the right alveolar nerve with the aid of an $8-\mathrm{cm}$ sural nerve graft to bridge the distance between nerve ends. Note that the need to cross the LABC from the left side of the neck to the right side of the face was not anticipated at the time the free flap was harvested; if a longer segment of LABC had been harvested from the donor site, the nerve graft may not have been necessary. The radial portion of the flap served to line the lower lip, while the ulnar portion formed the external lower lip and chin.

The patient's wounds were closed without complication (Fig. 2D). Two months postoperatively, the patient was orally competent, had good mandibular function, and had acceptable cosmesis (Fig. 2E, F, and G). She recovered protective sensation of the lower lip. Two and a half months after the procedure, she underwent a minor sequestrectomy and closure of a small oral cutaneous fistula. One month later, cancellous bone graft was placed to augment the free 


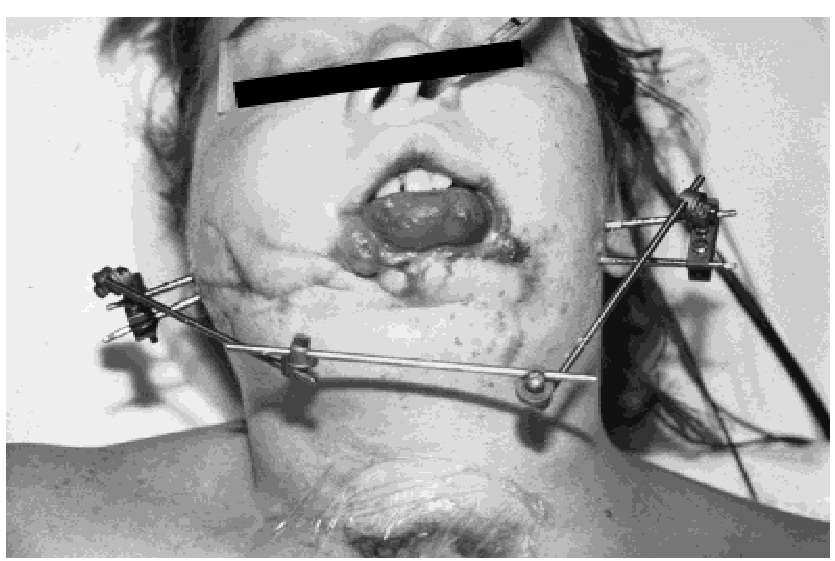

A

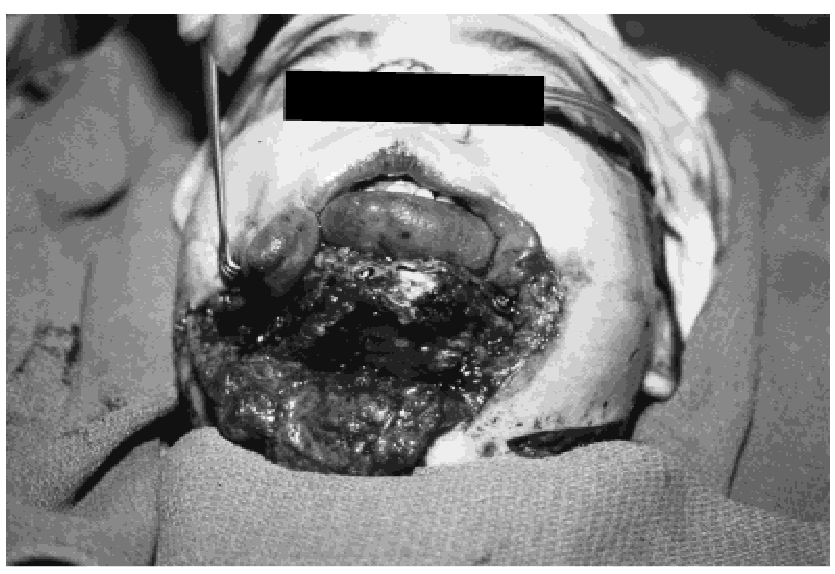

C

Figure 2. A: Preoperative frontal view of patient 2. Soft-tissue closure had been achieved by advancing neck and cheek skin into lower lip and chin defects. B: Preoperative profile demonstrating mandibular defect. C: Fibula osteocutaneous flap in place. Note extensive lower lip and chin defects, similar to those of patient 1. (Continued on the overleaf.)

fibula for subsequent osteo-integrated implants. A tongue flap was used to reconstruct the vermillion of the lower lip. Four years postoperatively, osteo-integrated implants were placed in the mandible. Currently, the patient is awaiting maturation of the osteo-integrated implants and placement of a dental prosthesis.

\section{DISCUSSION}

Although most head and neck reconstructions can be accomplished with a single free-tissue transfer, defects of massive size or involving diverse tissues may warrant the use of double free-flap reconstruction. Fulfilling both criteria, our two cases illustrate the utility of double free-flap reconstruction for the simultaneous one-stage reconstruction of two major defects: the entire lower lip and chin and the mandible.

The advantages of the fibula osteoseptocutaneous free

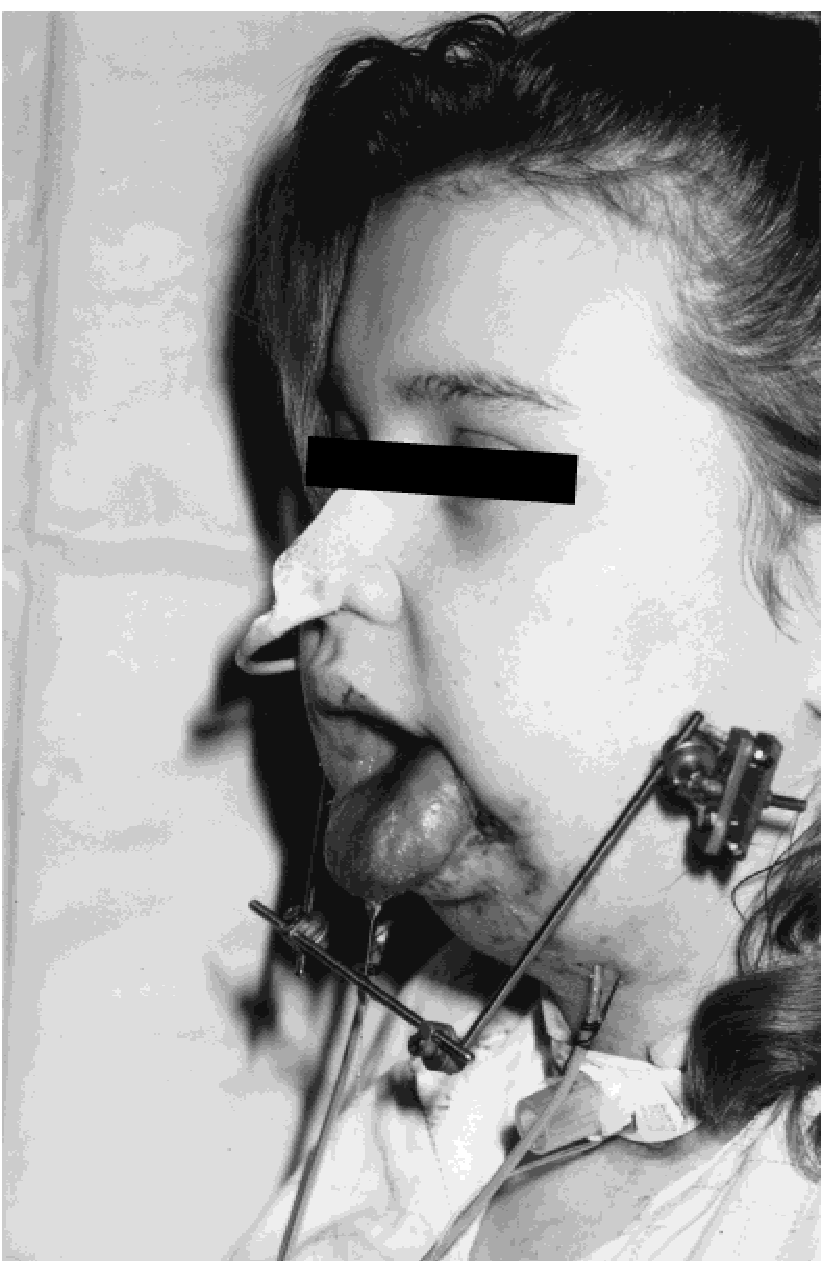

B

flap for the reconstruction of central mandibular defects have made it a workhorse for this purpose. ${ }^{3-5}$ However, in these two cases, a fibula osteoseptocutaneous flap alone would not have provided enough tissue to restore the lower lip and chin, and a fibular skin paddle would not have contained the vascularized palmaris longus tendon for functional reconstruction of the lower lip. By using the radial forearm-palmaris longus tendon composite flap to reconstruct the lower lip and chin, ${ }^{6}$ we were able to effect a superior functional and aesthetic reconstruction of all missing structures. Certainly, it would have been possible to provide adequate bone and soft tissue to fill the defect with an iliac crest osteocutaneous flap $^{7,8}$ or a multipaddle subscapular artery flap. ${ }^{9}$ Although the choice of these alternative donor sites may have provided some advantages, such as a better skin color match in the case of a scapular flap or an improved ability to utilize osteo-integrated dental implants for the iliac crest, neither of these approaches would 


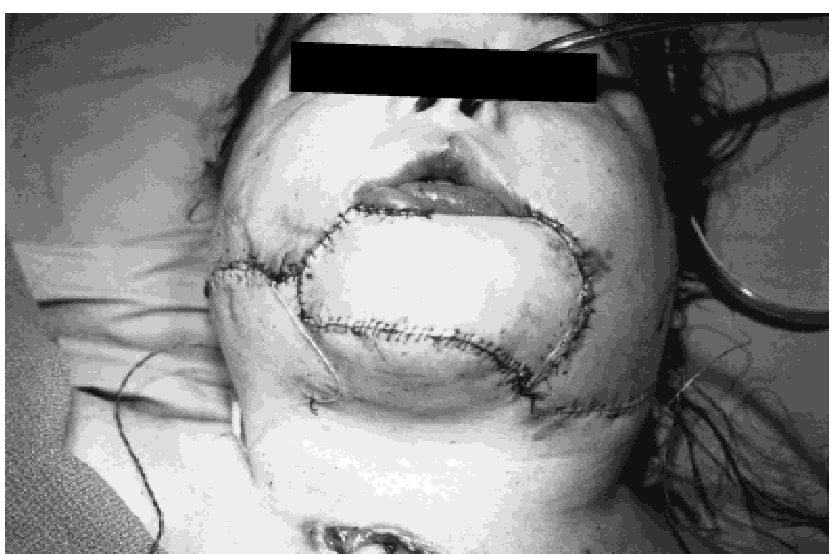

D

Figure 2. (Continued). D: Immediate postoperative view, with cutaneous incisions closed. E: Late postoperative frontal view.

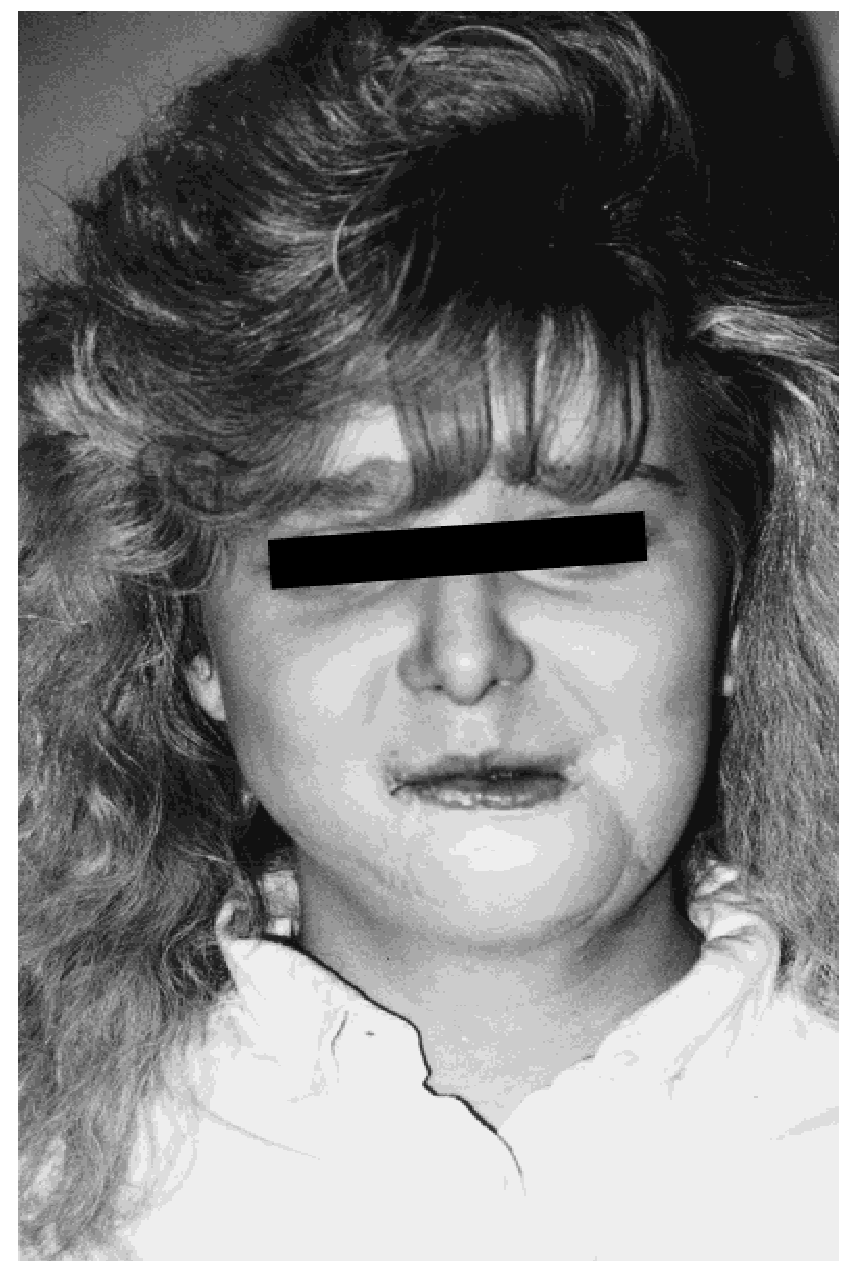

E

However, in both patients, two sets of recipient vessels were readily available in the neck, and the risks and technical difficulty associated with serial linkage of two free flaps mitigated against this approach. When recipient vessels are at a premium in the neck, however, sequential linkage of the fibula and radial forearm flap would allow this reconstruction to proceed even if only one recipient artery and vein were available.

In future cases, we would strongly consider several refinements that may have improved the results of these reconstructions. In both patients, it was our intention to reinnervate the radial forearm flap to provide a sensate lower lip. In Case 1, this became impossible because the ablative surgery destroyed both alveolar nerves far back into the mandibular canal and we were unwilling to compromise our bony reconstruction by resecting additional mandible to gain access to the alveolar nerves. In Case 2, a nerve graft was necessary to accomplish this goal because the segment of LABC harvested was too short to reach the recipient nerve. In both cases, appropriate preoperative planning may have allowed a primary coaptation between the LABC and 


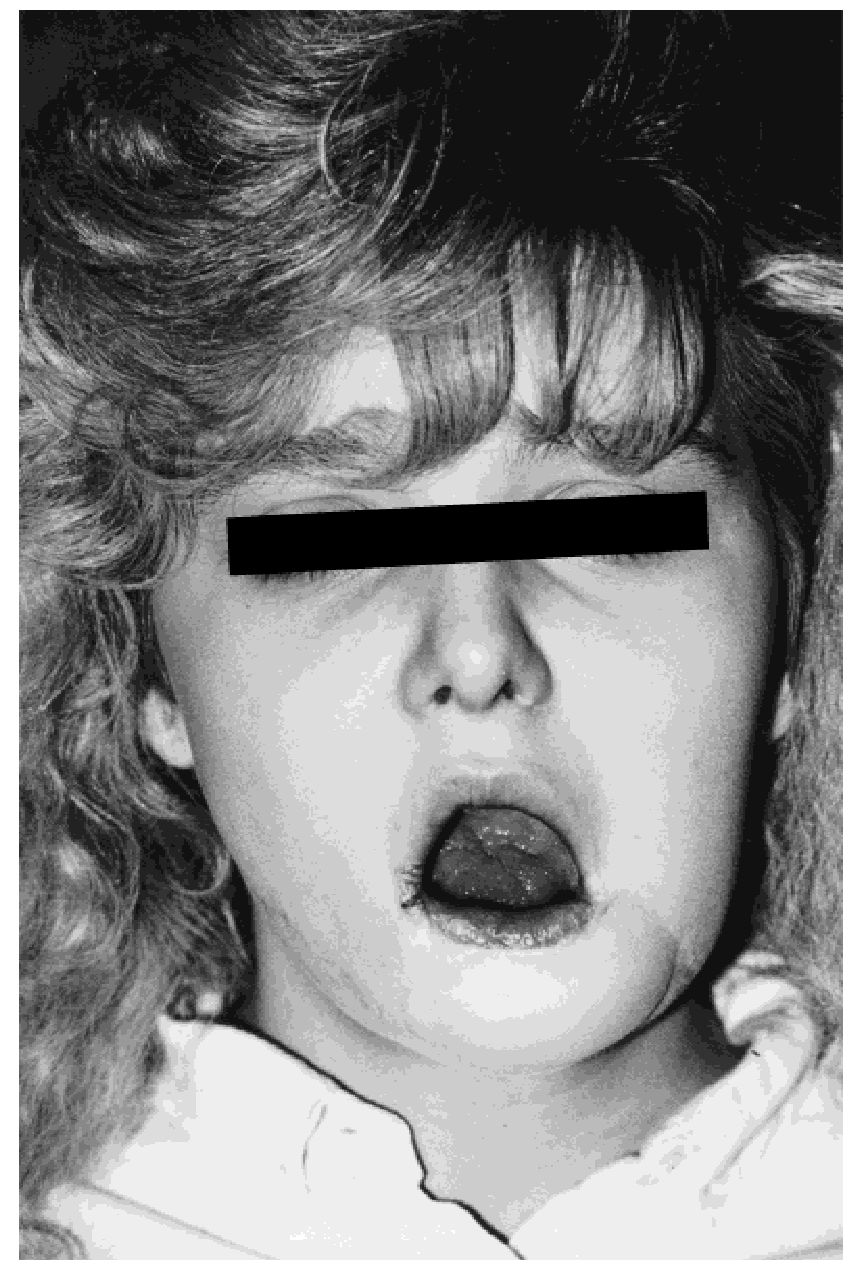

$\mathbf{F}$

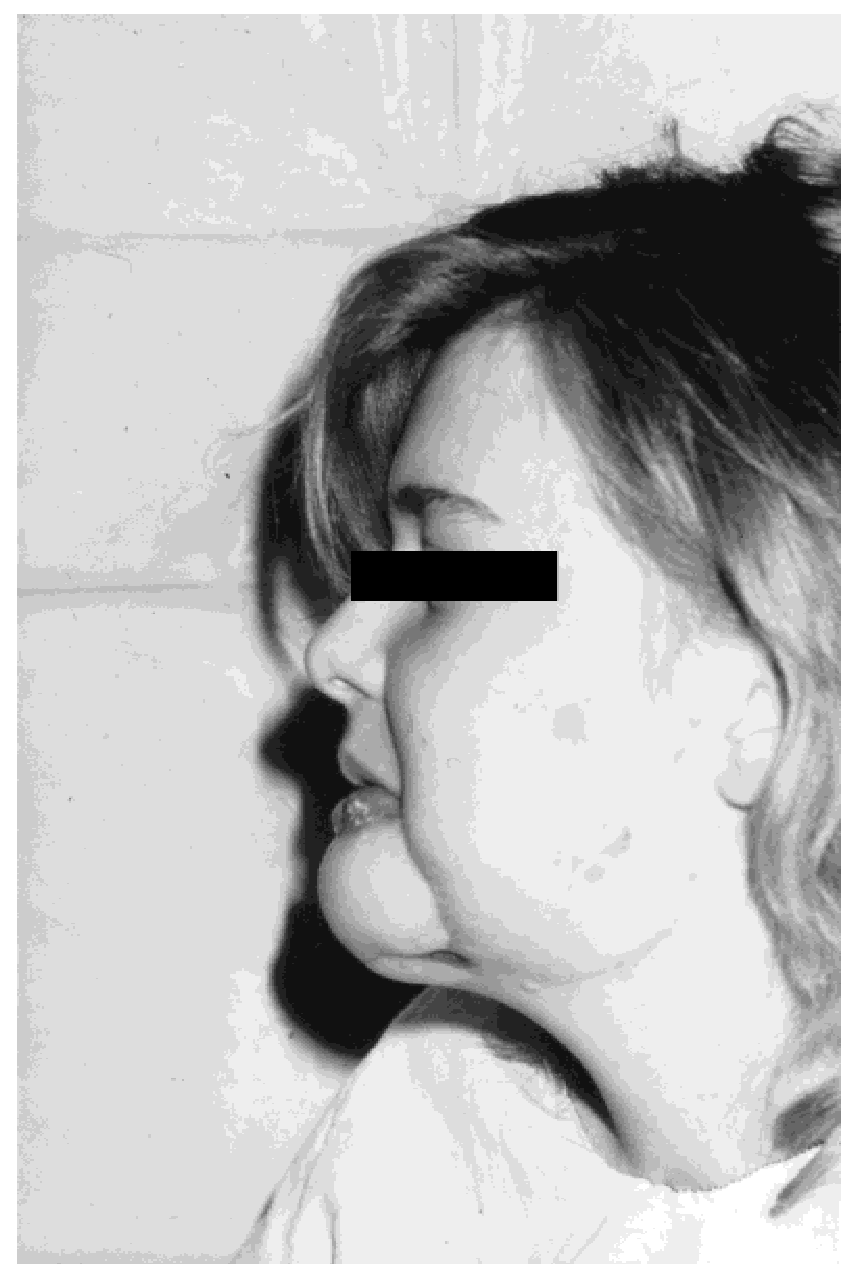

G

Figure 2. (Continued). F: Late postoperative frontal view demonstrating mandibular opening. G: Late profile view, demonstrating restoration of the chin.

an ipsilateral alveolar nerve. In addition, the functional reconstruction of the mandible may have been improved by reinserting the mandibular depressors on the inferior border of the neomandible. Lastly, in Case 1, the reconstruction of the labiobuccal sulcus may have been improved by utilizing a larger intraoral skin paddle or by suspension of the palmaris longus tendon not only to the modioli, but to multiple sites in the cheek as well.

In summary, two patients are presented who underwent reconstruction of total lower lip, chin, and mandible defects. Although double free-flap reconstruction is indicated only in truly massive or complex defects, the cases presented here illustrate the utility of this approach in addressing this unusual reconstructive challenge.

\section{ACKNOWLEDGMENTS}

The authors gratefully acknowledge the other attending physicians who assisted with the surgical care of these pa- tients: Riley Rees, M.D., E. Douglas Newton, M.D., James Ferraro, M.D., and John Pierce, D.D.S.

\section{REFERENCES}

1. Schusterman MA, Miller MJ, Reece GP, Kroll SS, Marchi M, Goepfert H: A single center's experience with 308 free flaps for repair of head and neck cancer defects. Plast Reconstr Surg 93:472-478, 1994.

2. Sanger JR, Yousif NJ, Matloub HS, Larson DL, Sewall SS: Reconstruction of lower third of face with three simultaneous free flaps. Plast Reconstr Surg 94:709-713, 1994.

3. Hidalgo DA: Fibula free flap: A new method of mandible reconstruction. Plast Reconstr Surg 84:71-79, 1989.

4. Hidalgo DA: Fibula free flap mandibular reconstruction. Clin Plast Surg 21:25-35, 1994.

5. Hidalgo DA, Rekow A: A review of 60 consecutive fibula free flap mandible reconstructions. Plast Reconstr Surg 96:585-596, 1995.

6. Sadove RC, Luce EA, McGrath PC: Reconstruction of the lower lip 
and chin with the composite radial forearm-palmaris longus free flap. Plast Reconstr Surg 88:209-214, 1991.

7. Boyd JB, Morris S, Rosen IB, Gullane P, Rotstein L, Freeman JL: The through-and-through oromandibular defect: Rationale for aggressive reconstruction. Plast Reconstr Surg 93:44-53, 1994.

8. Boyd JB: The place of the iliac crest in vascularized oromandibular reconstruction. Microsurgery 15:250-256, 1994.

9. Thomas A, Archibald S, Payk I, Young JE: The free medial scapular osteofasciocutaneous flap for head and neck reconstruction. Br J Plast Surg 44:477-482, 1991.

10. Sanger JR, Matloub HS, Yousif NJ: Sequential connection of flaps: A logical approach to customized mandibular reconstruction. Am J Surg 160:402-404, 1990.

11. Wells MD, Luce EA, Edwards AL, Vasconez HC, Sadove RC, Bouzaglou S: Sequentially linked free flaps in head and neck reconstruction. Clin Plast Surg 21:59-67, 1994. 